\title{
Theory with a unique thermal effective potential
}

\author{
Marcelo Hott* \\ UNESP, Campus de Guaratinguetá, P.O. Box 205, 12500-000, Guaratinguetá, São Paulo, Brazil \\ Georgios Metikas ${ }^{\dagger}$ \\ Abteilung für Quantenphysik, Universität Ulm, 11 Albert-Einstein-Allee, Ulm D-89069, Germany \\ and Theoretical Physics, University of Oxford, 1 Keble Road, Oxford OX1 3NP, United Kingdom
}

(Received 4 December 2001; published 9 April 2002)

\begin{abstract}
We show that for the pion-nucleon theory the thermal bubble graph is analytic at the origin of the momentum-frequency space, although the internal propagators in the loop have the same mass. This means that, for this theory, the thermal effective potential is uniquely defined. We then examine how a slight modification of the interaction term results in a theory for which the thermal bubble graph displays the usual nonanalyticity at the origin and the thermal effective potential is not uniquely defined.
\end{abstract}

DOI: $10.1103 /$ PhysRevD.65.085043

PACS number(s): 11.10.Wx

\section{INTRODUCTION}

It is well known that for most theories at finite temperature the self-energy displays a nonanalytic behavior at the origin of the momentum-frequency space [1-4]. This nonanalyticity manifests itself in a difference between the $\left\{q_{0}\right.$ $\rightarrow 0,|\mathbf{q}| \rightarrow 0\}$ and $\left\{|\mathbf{q}| \rightarrow 0, q_{0} \rightarrow 0\right\}$ limits of the self-energy, where $q_{0}$ and $\mathbf{q}$ are the components of the external momentum $q_{\mu}=\left(q_{0}, \mathbf{q}\right)$ and the component listed first goes to zero first. Consider for example a QED plasma at finite temperature. The first limit is associated with the screening of static electric fields by the plasma and gives a leading order approximation of the Debye mass which is a pole of the photon propagator and plays the role of the inverse screening length. The second limit has been used for the calculation of the plasma frequency [5-7]. One may argue that the two limits must differ since they refer to different physics [6].

This phenomenon was first pointed out in the BCS theory context by Abrahams and Tsuneto [8]. Later it was also seen to appear in a wide range of theories, for example in thermal QED $[9,10]$ and in thermal three-dimensional QED $\left(\mathrm{QED}_{3}\right)$ [11]. In thermal QCD it occurs in the gluon $[7,12]$ and in the massless quark self-energy $[13,14]$. Furthermore, it appears in all one-loop diagrams that have zero or two external massless quarks and any number of external gluons $[15,16]$. The problem is also present in the graviton self-energy $[17,18]$ and in higher-order graviton diagrams [19]. Even in the much simpler case of interacting scalars the nonanalyticity of the self-energy persists $[1,20-22]$.

The nonanalyticity of the thermal self-energy affects also the uniqueness of the thermal effective potential. When the standard, nonperturbative method of [23] is used at finite temperature, the resulting effective potential coincides with only one way of approaching the singularity of the selfenergy at the origin. In order to take into account the nonanalyticity, one can do perturbation over a weak coupling and then, provided the background field is slowly varying in

\footnotetext{
*Email address: hott@feg.unesp.br

${ }^{\dagger}$ Email address: george.metikas@physik.uni-ulm.de
}

space and time, one can apply derivative expansion techniques. The resulting effective potential will not be uniquely defined in this case [22].

The reason for this behavior is that temperature effects give rise to Landau terms in the self-energy and these are responsible for the development of a new branch cut in the complex plane of the external momenta with a branch point at the origin, besides the usual one which is already present at zero temperature $[4,24]$. The usual branch cut exists for

$$
s=q_{0}^{2}-|\mathbf{q}|^{2} \geqslant 4 m^{2}
$$

and there is a new branch point at

$$
s=q_{0}^{2}-|\mathbf{q}|^{2} \leqslant 0 .
$$

An interesting remark is that, whenever the internal propagators in a typical loop have different masses, the selfenergy is analytic at the origin [25]. In this nondegenerate mass case the usual branch cut is

$$
s \geqslant\left(m_{1}+m_{2}\right)^{2}
$$

and the new one is

$$
-\left|m_{1}^{2}-m_{2}^{2}\right| \leqslant s \leqslant\left(m_{1}-m_{2}\right)^{2}
$$

where $m_{1}$ and $m_{2}$ are the masses of the particles in the internal loop. The new branch point is not at the origin anymore and the problem disappears from this point, allowing thus the definition of a unique effective potential.

For the degenerate mass case, attempts have been made to find some way of circumventing the nonanalyticity of the self-energy at the origin of the momentum-frequency space $[26,27]$. However, Weldon in [1] showed that the analytic results of $[26,27]$ were an artifact of not taking into account certain subtleties of Feynman parametrization at finite temperature.

Here we present a theory which exhibits a new and unexpected feature: it has self-energy which is genuinely analytic 
at the origin, although the mass is degenerate. The theory is physically meaningful (the pion-nucleon model) and is the only known case where a thermal effective potential can be defined uniquely. Furthermore we discuss how subtle this new feature is and how the nonanalyticity can develop with the slightest change in the interaction term.

\section{A NEW CASE}

\section{A. Commuting limits} [28])

We consider the pion-nucleon model (see for example

$$
L[\bar{\psi}, \psi, \phi]=\bar{\psi}(i \not b-m) \psi-i g \bar{\psi} \gamma_{5} \psi \phi+L_{0}[\phi]
$$

where $\psi$ is a fermion and $\phi$ a boson. $L_{0}[\phi]$ is the free Klein-Gordon Lagrangian for the boson.

Integrating out the fermions, we obtain an effective action for the bosons. The one-loop contribution to this effective action is

$$
\Gamma_{e f f}[\phi]=-i \ln \frac{\operatorname{Det}\left[i S^{-1}[\phi]\right]}{\operatorname{Det}\left[i S^{-1}\right]}
$$

where $i S^{-1}[\phi]$ and $i S^{-1}$ are matrices whose elements in coordinate representation are

$$
\begin{aligned}
\left\langle x\left|i S^{-1}\right| y\right\rangle & =\left(i \partial_{x}-m\right) \delta(x-y) \\
\left\langle x\left|i S^{-1}[\phi]\right| y\right\rangle & =\left[i \partial_{x}-m-i g \gamma_{5} \phi(x)\right] \delta(x-y) .
\end{aligned}
$$

Since the bosonic field depends on the coordinates, the functional determinant $\operatorname{Det}\left[i S^{-1}[\phi]\right]$ in Eq. (2) is not straight- forward to calculate; the matrix whose functional determinant we want to evaluate is not diagonal in momentum or in coordinate space. However, progress can be made if we rewrite Eq. (2) as

$$
\Gamma_{e f f}[\phi]=-i \operatorname{Tr} \ln \left[1-g \gamma_{5} \phi(\hat{x}) S(\hat{p})\right]
$$

Now we expand the above expression in powers of the coupling constant and show that the leading contribution to the one-loop effective action is

$$
\Gamma^{(2)}=\frac{i g^{2}}{2} \int \frac{d^{4} q}{(2 \pi)^{4}} \widetilde{\phi}(-q) i \Pi(q) \widetilde{\phi}(q)
$$

where $\widetilde{\phi}(q)$ is the Fourier transformation of $\phi(x)$ and

$$
i \Pi(q)=\int \frac{d^{4} k}{(2 \pi)^{4}} \operatorname{tr}\left[\gamma_{5} \frac{1}{k+q-m} \gamma_{5} \frac{1}{k-m}\right] .
$$

We note that $i \Pi(q)$ is just the self-energy bubble diagram for the boson which, after performing the trace, is given by

$$
i \Pi(q)=-4 \int \frac{d^{4} k}{(2 \pi)^{4}} \frac{k^{2}+k^{\mu} q_{\mu}-m^{2}}{\left[(k+q)^{2}-m^{2}\right]\left[k^{2}-m^{2}\right]} .
$$

This is a typical diagram that usually has a nonanalytic behavior in the limit of vanishing external momenta but we will show that this is not the case here.

Applying the usual finite temperature techniques to Eq. (6), we find the following expression for the thermal bubble diagram:

$$
\begin{aligned}
\Pi\left(q_{0},|\mathbf{q}|\right)= & \int \frac{d^{3} \mathbf{k}}{(2 \pi)^{3}} \frac{1}{2 \omega \Omega}\left\{2 \omega\left[\tanh \frac{\beta\left(\Omega+q_{0}\right)}{2}+\tanh \frac{\beta\left(\Omega-q_{0}\right)}{2}\right]+\frac{1}{\Omega+\omega-q_{0}}\left[\left[\omega q_{0}+\mathbf{k q}\right] \tanh \frac{\beta \omega}{2}\right.\right. \\
& \left.+\left[q_{0}^{2}-\Omega q_{0}+\mathbf{k q}\right] \tanh \frac{\beta\left(\Omega-q_{0}\right)}{2}\right]+\frac{1}{\Omega+\omega+q_{0}}\left[\left[-\omega q_{0}+\mathbf{k q}\right] \tanh \frac{\beta \omega}{2}+\left[q_{0}^{2}+\Omega q_{0}+\mathbf{k q}\right] \tanh \frac{\beta\left(\Omega+q_{0}\right)}{2}\right] \\
& +\frac{1}{\Omega-\omega+q_{0}}\left[\left[\omega q_{0}+\mathbf{k q}\right] \tanh \frac{\beta \omega}{2}-\left[q_{0}^{2}+\Omega q_{0}+\mathbf{k q}\right] \tanh \frac{\beta\left(\Omega+q_{0}\right)}{2}\right]+\frac{1}{\Omega-\omega-q_{0}}\left[\left[-\omega q_{0}+\mathbf{k q}\right] \tanh \frac{\beta \omega}{2}\right. \\
& \left.\left.-\left[q_{0}^{2}-\Omega q_{0}+\mathbf{k q}\right] \tanh \frac{\beta\left(\Omega-q_{0}\right)}{2}\right]\right\}
\end{aligned}
$$

where

$$
\omega=\sqrt{\mathbf{k}^{2}+m^{2}} \quad \Omega=\sqrt{(\mathbf{k}+\mathbf{q})^{2}+m^{2}} \quad q_{0}=i \frac{2 \pi n}{\beta}, \quad n=\text { integer. }
$$

From the above definition of $q_{0}$ follows that

$$
e^{\beta q_{0}}=1
$$

and consequently $q_{0}$ disappears from all the hyperbolic tangents of Eq. (7): 


$$
\begin{aligned}
\Pi\left(q_{0},|\mathbf{q}|\right)= & \int \frac{d^{3} \mathbf{k}}{(2 \pi)^{3}} \frac{1}{2 \omega \Omega}\left\{4 \omega \tanh \frac{\beta \Omega}{2}+\frac{1}{\Omega+\omega-q_{0}}\left[\left[\omega q_{0}+\mathbf{k q}\right] \tanh \frac{\beta \omega}{2}+\left[q_{0}^{2}-\Omega q_{0}+\mathbf{k q}\right] \tanh \frac{\beta \Omega}{2}\right]\right. \\
& +\frac{1}{\Omega+\omega+q_{0}}\left[\left[-\omega q_{0}+\mathbf{k q}\right] \tanh \frac{\beta \omega}{2}+\left[q_{0}^{2}+\Omega q_{0}+\mathbf{k q}\right] \tanh \frac{\beta \Omega}{2}\right] \\
& +\frac{1}{\Omega-\omega+q_{0}}\left[\left[\omega q_{0}+\mathbf{k q}\right] \tanh \frac{\beta \omega}{2}-\left[q_{0}^{2}+\Omega q_{0}+\mathbf{k q}\right] \tanh \frac{\beta \Omega}{2}\right]+\frac{1}{\Omega-\omega-q_{0}} \\
& \left.\times\left[\left[-\omega q_{0}+\mathbf{k q}\right] \tanh \frac{\beta \omega}{2}-\left[q_{0}^{2}-\Omega q_{0}+\mathbf{k q}\right] \tanh \frac{\beta \Omega}{2}\right]\right\} .
\end{aligned}
$$

In the above expression (10), the last two terms are the Landau terms whose denominators vanish when the frequency and momentum are set to zero. We will be referring to the rest of the terms in Eq. (10) as the regular terms. The denominators of the regular terms do not vanish when the frequency and the momentum are set to zero. We also note that the Landau terms vanish at zero temperature and the only contribution left comes from the regular terms.

We analytically continue Eq. (10) to Minkowski space by letting the frequency $q_{0}$ be real. In fact, we should add to the real $q_{0}$ an infinitesimal imaginary part $i \epsilon$ whose sign determines whether we are evaluating advanced, retarded or Feynmann self-energies. Let us neglect for the moment this infinitesimal imaginary part; we will show later that its presence does not affect the limits we are calculating.

Now we can have a first indication that the two successive limits $\left\{q_{0} \rightarrow 0,|\mathbf{q}| \rightarrow 0\right\}$ and $\left\{|\mathbf{q}| \rightarrow 0, q_{0} \rightarrow 0\right\}$ coincide for the thermal bubble (10) of the pion-nucleon model, which thus does not display the usual nonuniqueness property

$$
\begin{aligned}
\Pi(0,|\mathbf{q}|)= & \int \frac{d^{3} \mathbf{k}}{(2 \pi)^{3}} \frac{1}{\omega \Omega}\left\{2 \omega \tanh \frac{\beta \Omega}{2}+\frac{\mathbf{k q}}{\Omega+\omega}\left[\tanh \frac{\beta \omega}{2}\right.\right. \\
& \left.\left.+\tanh \frac{\beta \Omega}{2}\right]+\frac{\mathbf{k q}}{\Omega-\omega}\left[\tanh \frac{\beta \omega}{2}-\tanh \frac{\beta \Omega}{2}\right]\right\} \\
& \longrightarrow \frac{\left|\mathbf{q}_{0}\right| \rightarrow 0}{\longrightarrow} \frac{1}{\pi^{2}} \int_{|m|}^{\infty} d \omega \sqrt{\omega^{2}-m^{2}} \tanh \frac{\beta \omega}{2}
\end{aligned}
$$

Similarly we find the other limit

$$
\begin{aligned}
\Pi\left(q_{0}, 0\right)= & \int \frac{d^{3} \mathbf{k}}{(2 \pi)^{3}} \frac{1}{2 \omega \Omega}\left\{4 \omega \tanh \frac{\beta \Omega}{2}+q_{0}^{2}\left[\frac{1}{2 \omega-q_{0}}\right.\right. \\
& \left.\left.+\frac{1}{2 \omega+q_{0}}\right] \tanh \frac{\beta \omega}{2}\right\} \\
& q_{0} \rightarrow 0 \\
& \longrightarrow \frac{1}{\pi^{2}} \int_{|m|}^{\infty} d \omega \sqrt{\omega^{2}-m^{2}} \tanh \frac{\beta \omega}{2}
\end{aligned}
$$

We conclude that the limits coincide. Moreover, the only term that contributes to the unique result is the first one in the integrand of Eq. (10) whereas the other two regular terms as well as the Landau terms vanish in this limit.

Of course, in the evaluation of the above limits, we interchanged the limits with both the integration over the momentum $|\mathbf{k}|$ and with the angular integration over $\theta$, so our conclusion is not entirely reliable so far. In principle, one should perform the integrations over $|\mathbf{k}|$ and $\theta$ first, and then take the limit. Unfortunately, in our case, the integration over $|\mathbf{k}|$ cannot be done analytically. However, we can perform the angular integration over $\theta$ before evaluating the limits.

Furthermore, instead of just taking the two successive limits $\left\{q_{0} \rightarrow 0,|\mathbf{q}| \rightarrow 0\right\}$ and $\left\{|\mathbf{q}| \rightarrow 0, q_{0} \rightarrow 0\right\}$, which in the momentum-frequency plane correspond to approaching the origin in the direction of one or the other axis, we can approach the origin through any other curve, for example in the direction of any straight line $q_{0}=a|\mathbf{q}|$, where $a$ can be any real number. If the limit $|\mathbf{q}| \rightarrow 0$ of $\Pi(a|\mathbf{q}|,|\mathbf{q}|)$ is independent of $a$, we will have a more general indication that the function is analytic at the origin, i.e., it does not depend on the way one approaches the origin $[1,25]$. Before doing so we recast Eq. (10) in a more convenient form by means of the transformation $\mathbf{k} \rightarrow-(\mathbf{k}+\mathbf{q})$ wherever the integrand contains $\tanh (\beta \Omega / 2)$ :

$$
\begin{aligned}
\Pi\left(q_{0},|\mathbf{q}|\right)= & \int \frac{d^{3} \mathbf{k}}{(2 \pi)^{3}}\left\{\frac{2}{\omega} \tanh \frac{\beta \omega}{2}+\left(q_{0}^{2}-\mathbf{q}^{2}\right) \tanh \frac{\beta \omega}{2}\right. \\
& \times \frac{1}{2 \omega \Omega}\left[\frac{1}{q_{0}+\Omega+\omega}-\frac{1}{q_{0}-\Omega-\omega}+\frac{1}{q_{0}+\Omega-\omega}\right.
\end{aligned}
$$

$$
\left.\left.-\frac{1}{q_{0}-\Omega+\omega}\right]\right\} \text {. }
$$

To perform the angular integration, we change variables from $\cos \theta$ to $\Omega$ and perform the integration over $\Omega$ :

$$
\begin{aligned}
\Pi\left(q_{0},|\mathbf{q}|\right)= & \frac{1}{\pi^{2}} \int_{|m|}^{\infty} d \omega \sqrt{\omega^{2}-m^{2}} \tanh \frac{\beta \omega}{2} \\
& +\frac{q_{0}^{2}-|\mathbf{q}|^{2}}{2|\mathbf{q}|} \int_{|m|(2 \pi)^{2}}^{\infty} \frac{d \omega}{\tanh \frac{\beta \omega}{2}} \\
& \times[L 1+L 2+L 3+L 4]
\end{aligned}
$$


where

$$
\begin{aligned}
& L 1\left(q_{0},|\mathbf{q}|\right)=\ln \frac{\Omega_{+}+\omega+q_{0}}{\Omega_{-}+\omega+q_{0}}, \quad L 2\left(q_{0},|\mathbf{q}|\right)=\ln \frac{\Omega_{+}+\omega-q_{0}}{\Omega_{-}+\omega-q_{0}} \\
& L 3\left(q_{0},|\mathbf{q}|\right)=\ln \frac{\Omega_{+}-\omega+q_{0}}{\Omega_{-}-\omega+q_{0}}, \quad L 4\left(q_{0},|\mathbf{q}|\right)=\ln \frac{\Omega_{+}-\omega-q_{0}}{\Omega_{-}-\omega-q_{0}}
\end{aligned}
$$

with

$$
\Omega_{+}=\sqrt{(|\mathbf{k}|+|\mathbf{q}|)^{2}+m^{2}}, \quad \Omega_{-}=\sqrt{(|\mathbf{k}|-|\mathbf{q}|)^{2}+m^{2}} .
$$

It is worth mentioning at this point that we still cannot perform analytically the integration over $|\mathbf{k}|$ (or equivalently over $\omega$ ) in Eq. (14). In other words, interchanging the order of the integrations over $|\mathbf{k}|$ and $\theta$ does not enable us to perform the $|\mathbf{k}|$ integration.

We proceed to the parametrization $q_{0}=a|\mathbf{q}|$ and examine the behavior of the self-energy as $|\mathbf{q}| \rightarrow 0$. The limits of the regular terms $L 1$ and $L 2$ are independent of $a$, as they should be. It is easy to see that

$$
\lim _{|\mathbf{q}| \rightarrow 0}\left\{\left(a^{2}-1\right)|\mathbf{q}|(L 1+L 2)\right\}=\left(a^{2}-1\right) 0 \ln 1=0 .
$$

What is quite unexpected is that, for this particular model, the contributions coming from the Landau terms, $L 3$ and $L 4$, vanish independently of $a$, that is

$$
\lim _{|\mathbf{q}| \rightarrow 0}\left\{\left(a^{2}-1\right)|\mathbf{q}|(L 3+L 4)\right\}=\left(a^{2}-1\right) 0 \ln 1=0 .
$$

This limit is less straightforward than Eq. (15), because the argument of the logarithm is an indefinite form as $|\mathbf{q}| \rightarrow 0$. However, we can Taylor-expand $\Omega_{+}$and $\Omega_{-}$around $|\mathbf{q}|$ $=0$ and cancel a power of $|\mathbf{q}|^{2}$ from numerator and denominator to see that

$$
\begin{aligned}
\lim _{|\mathbf{q}| \rightarrow 0}(L 3+L 4)= & \lim _{|\mathbf{q}| \rightarrow 0} \ln \frac{\left(\Omega_{+}-\omega\right)^{2}-a^{2}|\mathbf{q}|^{2}}{\left(\Omega_{-}-\omega\right)^{2}-a^{2}|\mathbf{q}|^{2}} \\
= & \lim _{|\mathbf{q}| \rightarrow 0} \ln \left\{\left(\left[\frac{|\mathbf{k}|}{\omega}+\frac{m^{2}|\mathbf{q}|}{2 \omega^{3}}\right.\right.\right. \\
& \left.\left.+O\left(q^{2}\right)\right]^{2}-a^{2}\right) /\left(\left[-\frac{|\mathbf{k}|}{\omega}+\frac{m^{2}|\mathbf{q}|}{2 \omega^{3}}\right.\right. \\
& \left.\left.\left.+O\left(q^{2}\right)\right]^{2}-a^{2}\right)\right\}=\ln 1=0
\end{aligned}
$$

\section{B. Imaginary parts}

If $q_{0}$ is made complex and continuous, the only poles or zeros of the sum of $L$ 's in Eq. (14) occur for $q_{0}$ on the real axis. It is perfectly appropriate to have singularities on the real axis. Thus the analytic extension of Eq. (14) is trivially obtained by letting $q_{0}$ be real. There are three self-energies on the real axis:

$$
\begin{gathered}
\Pi_{R}\left(q_{0},|\mathbf{q}|\right)=\Pi\left(q_{0}+i \epsilon,|\mathbf{q}|\right), \\
\Pi_{A}\left(q_{0},|\mathbf{q}|\right)=\Pi\left(q_{0}-i \epsilon,|\mathbf{q}|\right), \\
\Pi_{F}\left(q_{0},|\mathbf{q}|\right)=\Pi\left(q_{0}+i \epsilon q_{0},|\mathbf{q}|\right),
\end{gathered}
$$

where $\epsilon \rightarrow 0^{+}$. The real parts of these self-energies coincide whereas the imaginary parts are related according to

$$
\operatorname{Im} \Pi_{R}=-\operatorname{Im} \Pi_{A}=\operatorname{sgn}\left(q_{0}\right) \operatorname{Im} \Pi_{F}
$$

where $\operatorname{sgn}\left(q_{0}\right)$ is the sign of the frequency $q_{0}$.

Following [1] we shall not concern ourselves with the Feynman self-energy. Using the fact that $\epsilon$ is infinitesimal, the real part of the self-energy can be shown to be

$$
\begin{aligned}
\operatorname{Re} \Pi\left(q_{0},|\mathbf{q}|\right)= & \frac{1}{\pi^{2}} \int_{|m|}^{\infty} d \omega \sqrt{\omega^{2}-m^{2}} \tanh \frac{\beta \omega}{2} \\
& +\frac{q_{0}^{2}-|\mathbf{q}|^{2}}{2|\mathbf{q}|} \int_{|m|}^{\infty} \frac{d \omega}{(2 \pi)^{2}} \tanh \frac{\beta \omega}{2} \\
& \times[\operatorname{Re} L 1+\operatorname{Re} L 2+\operatorname{Re} L 3+\operatorname{Re} L 4]
\end{aligned}
$$

where

$$
\begin{aligned}
& \operatorname{Re} L 1\left(q_{0},|\mathbf{q}|\right)=\ln \left|\frac{\Omega_{+}+\omega+q_{0}}{\Omega_{-}+\omega+q_{0}}\right|, \\
& \operatorname{Re} L 2\left(q_{0},|\mathbf{q}|\right)=\ln \left|\frac{\Omega_{+}+\omega-q_{0}}{\Omega_{-}+\omega-q_{0}}\right|, \\
& \operatorname{Re} L 3\left(q_{0},|\mathbf{q}|\right)=\ln \left|\frac{\Omega_{+}-\omega+q_{0}}{\Omega_{-}-\omega+q_{0}}\right|, \\
& \operatorname{Re} L 4\left(q_{0},|\mathbf{q}|\right)=\ln \left|\frac{\Omega_{+}-\omega-q_{0}}{\Omega_{-}-\omega-q_{0}}\right| .
\end{aligned}
$$

The only difference between Eqs. (19) and (14) is that in the former the absolute value of the arguments of the logarithms is taken. It is easy to show that the limit of the real part of the regular terms is

$$
\lim _{|\mathbf{q}| \rightarrow 0}\left\{\left(a^{2}-1\right)|\mathbf{q}|(\operatorname{Re} L 1+\operatorname{Re} L 2)\right\}=0,
$$

and the limit of the real part of the Landau terms is

$$
\lim _{|\mathbf{q}| \rightarrow 0}\left\{\left(a^{2}-1\right)|\mathbf{q}|(\operatorname{Re} L 3+\operatorname{Re} L 4)\right\}=0
$$

These limits coincide with the limits of Eqs. (15) and (16). The reason is that, as $|\mathbf{q}| \rightarrow 0$, the arguments of the corresponding logarithms in Eqs. (15) and (16) tend to the positive number 1 for any real $a$. 
We note that the real part of the self-energy is even under $q_{0} \rightarrow-q_{0}$, since it can be written as a function of $q_{0}^{2}$, if we combine the logarithms. It is also even under $\mathbf{q} \rightarrow-\mathbf{q}$, since it depends only on $|\mathbf{q}|$.

Now we turn to the imaginary part which we calculate making use of the following form of the delta function:

$$
\delta(x)=\frac{i}{2 \pi} \lim _{\epsilon \rightarrow 0^{+}}\left[\frac{1}{x+i \epsilon}-\frac{1}{x-i \epsilon}\right] .
$$

The imaginary part is

$$
\begin{aligned}
\operatorname{Im} \Pi_{R}= & -\operatorname{Im} \Pi_{A}=\frac{1}{2} \int \frac{d^{3} \mathbf{k}}{(2 \pi)^{2}} \frac{1}{2 \omega \Omega}\left(q_{0}^{2}-\mathbf{q}^{2}\right) \tanh \frac{\beta \omega}{2} \\
& \times\left[\delta\left(q_{0}+\Omega+\omega\right)-\delta\left(q_{0}-\Omega-\omega\right)+\delta\left(q_{0}+\Omega-\omega\right)\right. \\
& \left.-\delta\left(q_{0}-\Omega+\omega\right)\right] .
\end{aligned}
$$

We note that it is odd under $q_{0} \rightarrow-q_{0}$. However it is even under $\mathbf{q} \rightarrow-\mathbf{q}$, because we can simultaneously change the integration variable $\mathbf{k} \rightarrow-\mathbf{k}$. This means that, unlike the real part, the imaginary part of the retarded or advanced thermal self-energy does not contribute to the effective action. As we can see from Eq. (4), the integrand of the effective action is $\widetilde{\phi}\left(q_{0}, \mathbf{q}\right) \widetilde{\phi}\left(-q_{0},-\mathbf{q}\right) \Pi\left(q_{0}, \mathbf{q}\right)$ and therefore the contribution $\widetilde{\phi}\left(q_{0}, \mathbf{q}\right) \widetilde{\phi}\left(-q_{0},-\mathbf{q}\right) \operatorname{Im} \Pi\left(q_{0}, \mathbf{q}\right)$ is odd under the combined transformations $q_{0} \rightarrow-q_{0}$ and $\mathbf{q} \rightarrow-\mathbf{q}$ and vanishes, when integrated over $d^{4} q$. As we are interested only in the effective action and the effective potential for the bosons, we will not concern ourselves with the imaginary part of the thermal bubble for the rest of this section. For a discussion of the physical significance of the imaginary part of the thermal bubble, see $[3,24]$.

\section{Effective potential}

The theory of this section is very special; although the Landau terms are not well-behaved at the origin of the momentum-frequency space, a unique effective potential up to second order in the coupling constant can be defined thanks to the kinetic term in the numerator of Eq. (13), namely $q_{0}^{2}-\mathbf{q}^{2}$. The one-loop, $g^{2}$ order contribution to the effective potential is

$$
\begin{aligned}
V_{e f f}^{(2)} & =\frac{g^{2}}{2} \operatorname{Re} \Pi(0,0) \phi^{2} \\
\operatorname{Re} \Pi(0,0) & =\frac{1}{\pi^{2}} \int_{|m|}^{\infty} d \omega \sqrt{\omega^{2}-m^{2}} \tanh \frac{\beta \omega}{2} .
\end{aligned}
$$

\section{THE USUAL CASE}

In this section we consider a model of fermions interacting with scalar bosons whose only difference from the one which we examined in the previous section is that its interaction term does not contain the $\gamma_{5}$ matrix

$$
L^{\prime}[\bar{\psi}, \psi, \phi]=\bar{\psi}(i \not b-m) \psi-i g \bar{\psi} \psi \phi+L_{0}[\phi]
$$

As we shall soon see, this simple modification of the interaction term has far-reaching consequences as far as the analytic properties of the thermal self-energy are concerned. Starting from Eq. (25) and following the procedure of the previous section we find that the one-loop effective action is

$$
\Gamma_{e f f}^{\prime}[\phi]=-i \operatorname{Tr} \ln [1-g \phi(\hat{x}) S(\hat{p})]
$$

and the self-energy bubble is given by

$$
i \Pi^{\prime}(q)=4 \int \frac{d^{4} k}{(2 \pi)^{4}} \frac{k^{2}+k^{\mu} q_{\mu}+m^{2}}{\left[(k+q)^{2}-m^{2}\right]\left[k^{2}-m^{2}\right]}
$$

which can be written as

$$
i \Pi^{\prime}(q)=-i \Pi(q)+i \Pi^{\prime \prime}(q),
$$

where

$$
i \Pi^{\prime \prime}(q)=4 \int \frac{d^{4} k}{(2 \pi)^{4}} \frac{2 m^{2}}{\left[(k+q)^{2}-m^{2}\right]\left[k^{2}-m^{2}\right]}
$$

As we saw in the previous section $\operatorname{Re} \Pi(a|\mathbf{q}|,|\mathbf{q}|)$ does not depend on $a$, when $|\mathbf{q}| \rightarrow 0$. We will see that $\operatorname{Re} \Pi^{\prime \prime}(a|\mathbf{q}|,|\mathbf{q}|)$ does. At finite temperature,

$$
\begin{aligned}
\Pi^{\prime \prime}\left(q_{0},|\mathbf{q}|\right)= & -m^{2} \int \frac{d^{3} \mathbf{k}}{(2 \pi)^{3}} \frac{1}{\omega \Omega}\left\{\left[\frac{1}{\Omega+\omega-q_{0}}+\frac{1}{\Omega-\omega+q_{0}}\right] \tanh \frac{\beta \omega}{2}+\left[\frac{1}{\Omega+\omega+q_{0}}+\frac{1}{\Omega-\omega-q_{0}}\right] \tanh \frac{\beta \omega}{2}\right. \\
& \left.+\left[\frac{1}{\Omega+\omega+q_{0}}-\frac{1}{\Omega-\omega+q_{0}}\right] \tanh \frac{\beta\left(\Omega+q_{0}\right)}{2}+\left[\frac{1}{\Omega+\omega-q_{0}}-\frac{1}{\Omega-\omega-q_{0}}\right] \tanh \frac{\beta\left(\Omega-q_{0}\right)}{2}\right\} .
\end{aligned}
$$

We now let the frequency $q_{0}$ be real. As in the previous section, we are not concerned with the infinitesimal imaginary parts. Interchanging the two successive limits with the integrations yields 


$$
\begin{aligned}
\Pi^{\prime \prime}(0,|\mathbf{q}|)= & -\int \frac{d^{3} \mathbf{k}}{(2 \pi)^{3}} \frac{1}{\omega \Omega}\left\{\frac{2 m^{2}}{\Omega+\omega}\left[\tanh \frac{\beta \omega}{2}+\tanh \frac{\beta \Omega}{2}\right]\right. \\
& \left.+\frac{2 m^{2}}{\Omega-\omega}\left[\tanh \frac{\beta \omega}{2}-\tanh \frac{\beta \Omega}{2}\right]\right\} \\
\stackrel{|\mathbf{q}| \rightarrow 0}{\longrightarrow} & -\frac{1}{\pi^{2}} \int_{|m|}^{\infty} d \omega \sqrt{\omega^{2}-m^{2}} \\
& \times\left\{\frac{m^{2}}{\omega^{2}} \tanh \frac{\beta \omega}{2}-\frac{\beta m^{2}}{2 \omega} \cosh ^{-2} \frac{\beta \omega}{2}\right\}
\end{aligned}
$$

and

$$
\begin{aligned}
\Pi^{\prime \prime}\left(q_{0}, 0\right) & =-\int \frac{d^{3} \mathbf{k}}{(2 \pi)^{3}} \frac{1}{\omega^{2}}\left\{\frac{2 m^{2}}{2 \omega-q_{0}}+\frac{2 m^{2}}{2 \omega+q_{0}}\right\} \tanh \frac{\beta \omega}{2} \\
& \longrightarrow-\frac{1}{\pi^{2}} \int_{|m|}^{\infty} d \omega \sqrt{\omega^{2}-m^{2}} \frac{m^{2}}{\omega^{2}} \tanh \frac{\beta \omega}{2} .
\end{aligned}
$$

The two successive limits of the thermal bubble do not commute. In order to better establish this conclusion, we perform the angular integration first and then evaluate the limits.

If we use Eq. (9) and apply the transformation $\mathbf{k} \rightarrow-(\mathbf{k}$ + q) to the terms containing $\tanh (\beta \Omega / 2)$, Eq. (28) reads

$$
\begin{aligned}
\Pi^{\prime \prime}\left(q_{0},|\mathbf{q}|\right)= & -2 m^{2} \int \frac{d^{3} \mathbf{k}}{(2 \pi)^{3}} \frac{1}{\omega \Omega} \tanh \frac{\beta \omega}{2}\left\{\frac{1}{q_{0}+\Omega+\omega}\right. \\
& \left.-\frac{1}{q_{0}-\Omega-\omega}+\frac{1}{q_{0}+\Omega-\omega}-\frac{1}{q_{0}-\Omega+\omega}\right\} .
\end{aligned}
$$

The transformation $\mathbf{k} \rightarrow-(\mathbf{k}+\mathbf{q})$ which was perfectly permissible in the previous section is not so here. The reason is that, if we interchange the limit $\left\{q_{0} \rightarrow 0,|\mathbf{q}| \rightarrow 0\right\}$ with the integrations, we see that, while in Eq. (28) the Landau terms are finite in this limit, in Eq. (31), that is after the transformation, the Landau terms diverge. These divergencies cancel out in the combination in which the Landau terms appear in Eq. (28), but once we split the terms of Eq. (28) and transform only the last two, the divergencies do not cancel each other anymore. Consequently, Eq. (31) is valid provided we do not take the limit $\left\{q_{0} \rightarrow 0,|\mathbf{q}| \rightarrow 0\right\}$ in the integral.

Performing the angular integration, setting $q_{0}=a|\mathbf{q}|$ and following the steps of the previous section yields the effective potential

$$
\begin{aligned}
\left(V_{e f f}^{(2)}\right)^{\prime \prime}= & \frac{g^{2}}{2} \operatorname{Re} \Pi^{\prime \prime}(0,0) \phi^{2} \\
\operatorname{Re} \Pi^{\prime \prime}(0,0)= & -\frac{1}{\pi^{2}} \int_{|m|}^{\infty} d \omega \sqrt{\omega^{2}-m^{2}} \tanh \frac{\beta \omega}{2} \\
& \times\left\{\frac{m^{2}}{\omega^{2}}+\frac{m^{4}}{\left(\omega^{2}-m^{2}\right) \omega^{2}-a^{2} \omega^{4}}\right\} .
\end{aligned}
$$

The value of the thermal bubble at the origin of the momentum-frequency space depends on how the origin is approached. For $a \rightarrow \infty$ we obtain the result of Eq. (30). We are still not allowed to take the limit $a \rightarrow 0$ into the integral, but after an integration by parts, we find, for $a<1$,

$$
\begin{aligned}
\operatorname{Re} \Pi^{\prime \prime}(0,0)= & -\frac{1}{\pi^{2}} \int_{|m|}^{\infty} d \omega \sqrt{\omega^{2}-m^{2}} \tanh \frac{\beta \omega}{2} \frac{m^{2}}{\omega^{2}} \\
& -\frac{1}{\pi^{2}}\left\{\left[G(\omega) \tanh \frac{\beta \omega}{2}\right]_{|m|}^{\infty}\right. \\
& \left.-\int_{|m|}^{\infty} d \omega G(\omega) \frac{\beta}{2} \cosh ^{-2} \frac{\beta \omega}{2}\right\}
\end{aligned}
$$

where

$$
\begin{aligned}
G(\omega)= & \frac{m^{2}}{2 \omega}\left\{2 \sqrt{\omega^{2}-m^{2}}+a \omega \ln \left[\frac{m+\sqrt{1-a^{2}} \omega}{m-\sqrt{1-a^{2}} \omega}\right.\right. \\
& \left.\left.\times \frac{\sqrt{1-a^{2}} m-\omega+a \sqrt{\omega^{2}-m^{2}}}{\sqrt{1-a^{2}} m+\omega+a \sqrt{\omega^{2}-m^{2}}}\right]\right\} .
\end{aligned}
$$

Now we may safely perform the $a \rightarrow 0$ limit to find the result of Eq. (29).

As in the previous section, the imaginary part does not contribute to the effective action. Therefore the total effective potential for the theory of this section is

$$
\begin{aligned}
\left(V_{e f f}^{(2)}\right)^{\prime} & =\frac{g^{2}}{2}\left[-\operatorname{Re} \Pi(0,0)+\operatorname{Re} \Pi^{\prime \prime}(0,0)\right] \phi^{2} \\
& = \begin{cases}-\frac{g^{2}}{2 \pi^{2}} \int_{|m|}^{\infty} d \omega \sqrt{\omega^{2}-m^{2}} \tanh \frac{\beta \omega}{2}\left\{1+\frac{m^{2}}{\omega^{2}}+\frac{m^{4}}{\left(\omega^{2}-m^{2}\right) \omega^{2}-a^{2} \omega^{4}}\right\} \phi^{2} & \text { if } a \neq 0, \\
-\frac{g^{2}}{2 \pi^{2}} \int_{|m|}^{\infty} d \omega \sqrt{\omega^{2}-m^{2}} \tanh \frac{\beta \omega}{2}\left\{1+\frac{m^{2}}{\omega^{2}}-\frac{\beta^{2} m^{2}}{\beta \omega \sinh \beta \omega}\right\} \phi^{2} & \text { if } a=0 .\end{cases}
\end{aligned}
$$


This effective potential is not uniquely defined, because it depends on $a$ which can take any real value. Comparing Eq. (6) to Eq. (27), we see that dropping $\gamma_{5}$ from the interaction resulted in changing the relative sign between the momentum terms and $m^{2}$ in the numerator. This slight change was enough to allow for the development of a self-energy which is nonanalytic at the origin.

\section{CONCLUSIONS}

We have shown that, in the pion-nucleon model, the effective potential for the bosonic field is unique at finite temperature. We have also shown that this is not true when the interaction term is slightly modified, the reason for that being the nonanalytic behavior which appears in the thermal bubble diagram.

A further consequence of the analyticity displayed by the pion-nucleon theory is that the Debye mass at leading order (11) and the plasma frequency at leading order (12) coincide. This is not the case for the modified theory as we can see from Eqs. (29) and (30).

The models we dealt with in Secs. II and III can be considered together to study chiral symmetry restoration at finite temperature for example in the Lurie model [29], the linear $\sigma$ model [30] in its broken chiral symmetry phase and in the
Nambu-Jona-Lasinio model [31] expressed in terms of auxiliary fields.

In general, with the exception of the pion-nucleon model discussed above and, possibly, other special cases, when finite temperature symmetry restoration is discussed by employing nonperturbative results for the effective potential, these may not match the perturbative results. The nonperturbative results, for example $[23,27]$, when expanded perturbatively, reproduce only the $a=0$ case of those calculated by means of perturbation theory. Therefore the question of symmetry restoration at finite temperature should be reanalyzed keeping in mind the nonanalyticity of certain graphs. Work on this and other related issues is in progress.

\section{ACKNOWLEDGMENTS}

The authors wish to thank Professor I.J.R. Aitchison for providing the opportunity for the commencement of this work as well as for numerous illuminating discussions. G.M. thanks Professor G. Alber for many insightful remarks on this and other related topics. M.H. thanks Dr. H. Caldas for helpful comments. M.H. was partially funded by FAPESP (São Paulo, Brazil). The work of G.M. was supported by the Deutsche Forschungsgemeinschaft within the Forschergruppe "Quantengase."
[1] H.A. Weldon, Phys. Rev. D 47, 594 (1993).

[2] J.I. Kapusta, Finite-Temperature Field Theory (Cambridge University Press, Cambridge, England, 1989).

[3] M. Le Bellac, Thermal Field Theory (Cambridge University Press, Cambridge, England, 1996).

[4] A. Das, Finite Temperature Field Theory (World Scientific, Singapore, 1997).

[5] J.D. Jackson, Classical Electrodynamics (Wiley, New York, 1975).

[6] K. Kajantie and J. Kapusta, Ann. Phys. (N.Y.) 160, 477 (1985).

[7] H.A. Weldon, Phys. Rev. D 26, 1394 (1982).

[8] E. Abrahams and T. Tsuneto, Phys. Rev. 152, 416 (1966).

[9] G. Baym, J.P. Blaizot, and B. Svetitsky, Phys. Rev. D 46, 4043 (1992).

[10] E. Petitgirard, Z. Phys. C 54, 673 (1992).

[11] I.J.R. Aitchison and J.A. Zuk, Ann. Phys. (N.Y.) 242, 77 (1995).

[12] O.K. Kalashnikov and V.V. Klimov, Sov. J. Nucl. Phys. 31, 699 (1980).

[13] V.V. Klimov, Sov. J. Nucl. Phys. 33, 934 (1981).
[14] H.A. Weldon, Phys. Rev. D 26, 2789 (1982).

[15] E. Braaten and R.D. Pisarski, Nucl. Phys. B337, 569 (1990).

[16] J. Frenkel and J.C. Taylor, Nucl. Phys. B334, 199 (1990).

[17] A. Rebhan, Nucl. Phys. B368, 479 (1992).

[18] A. Rebhan, Nucl. Phys. B351, 706 (1991).

[19] J. Frenkel and J.C. Taylor, Z. Phys. C 49, 515 (1991).

[20] Y. Fujimoto and H. Yamada, Z. Phys. C 37, 265 (1988).

[21] T.S. Evans, Z. Phys. C 36, 153 (1987).

[22] A. Das and M. Hott, Phys. Rev. D 50, 6655 (1994).

[23] L. Dolan and R. Jackiw, Phys. Rev. D 9, 3320 (1974).

[24] H.A. Weldon, Phys. Rev. D 28, 2007 (1983).

[25] P. Arnold, S. Vokos, P. Bedaque, and A. Das, Phys. Rev. D 47, 4698 (1993).

[26] P.F. Bedaque and A. Das, Phys. Rev. D 45, 2906 (1992).

[27] P.S. Gribosky and B.R. Holstein, Z. Phys. C 47, 205 (1990).

[28] P. Roman, Introduction to Quantum Field Theory (Wiley, New York, 1969).

[29] Wang Enke and Li Jiarong, Phys. Rev. D 44, 3680 (1991).

[30] M. Gell-Mann and M. Levy, Nuovo Cimento 16, 705 (1960).

[31] Y. Nambu and G. Jona-Lasinio, Phys. Rev. 122, 345 (1961). 